\title{
Molecular Imaging in the Era of Precision Medicine: Paraganglioma as a Template for Understanding Multiple Levels of Analysis
}

\author{
David Taïeb ${ }^{1}$, Dimitris Visvikis ${ }^{2}$, Rodney J. Hicks ${ }^{3}$, and Karel Pacak ${ }^{4}$ \\ ${ }^{I}$ Department of Nuclear Medicine, La Timone University Hospital, CERIMED, Aix-Marseille Univ, Marseille, France; ${ }^{2}$ LaTIM, \\ INSERM, UMR 1101, Univ Brest, Brest, France; ${ }^{3}$ The Sir Peter MacCallum Department of Oncology, the University of Melbourne, \\ Melbourne 3000 Victoria, Australia; and ${ }^{4}$ Section on Medical Neuroendocrinology, Eunice Kennedy Shriver National Institute of \\ Child Health \& Human Development (NICHD), National Institutes of Health, Bethesda, Maryland
}

$\mathbf{N}$ uclear (molecular) imaging plays a central role in oncology by allowing in vivo visualization of molecular dysregulation as well as overexpression of certain cell membrane transporters, receptors, or tumor antigens (1). There are multiple ways to analyze data emerging from this technology: descriptive-which occurs in routine clinical practice; quantitative-or radiomics, which provides prognostic, predictive, and companion biomarkers; integrative-which is linked with molecular genetics; and conceptual-which aims to advance and expand the nosologic classification of cancers. Rapid advances in technology and artificial intelligence (AI) are already affecting these aspects and leading to a convergence of each dimension. Paragangliomas (PGL) and pheochromocytomas (PHEO or adrenal PGL), collectively referred to as PPGL, are neuroendocrine neoplasms arising from endocrine cells of neural crest origin (mostly from multifated Schwann cell precursors). These tumors are caused by inherited genetic mutations more often than other neuroendocrine tumors. They represent an outstanding example of how molecular imaging is connected to tumor biology.

The first level of analysis is positioned at an anatomic, biochemical, and physiologic scale, with a primary purpose of lesion detection. Nuclear physicians should state a likely or preferred differential diagnosis for observed foci of uptake considered to be abnormal and integrate these findings in a specific clinical setting. The added value of molecular imaging compared with classic radiologic imaging relies on the use of various specific radiopharmaceuticals that are avidly taken up by tumors. This results in a high tumor-tobackground uptake ratio, which is optimal for tumor detection (sensitivity), but is also associated with a high positive predictive value, which avoids the relatively high rate of false-positive results that are generally associated with very sensitive anatomic imaging modalities. The use of hybrid imaging means that all of the complementary anatomic information required for surgical planning is still potentially assessable. For PPGL, a PET/CT report should include the following information: confirmation of diagnosis of PPGL in doubtful

Received Oct. 30, 2019; revision accepted Jan. 10, 2020.

For correspondence or reprints contact: David Taïeb, Department of Nuclear Medicine, La Timone University Hospital, European Center for Research in Medical Imaging, Aix-Marseille University, 264 rue Saint-Pierre, 13385 Marseille, France.

E-mail: david.taieb@ap-hm.fr

Published online Mar. 13, 2020.

COPYRIGHT (C) 2020 by the Society of Nuclear Medicine and Molecular Imaging. DOI: 10.2967/jnumed.119.239095 situations; evaluation of locoregional extension, especially in head and neck PGL, which together with anatomic imaging can predict surgical outcome; detection of tumor multiplicity at each anatomic level (i.e., head and neck, mediastinum, adrenal, abdomen, and pelvis); and detection of potential metastases (malignancy being defined only by the presence of metastatic lesions at sites in which chromaffin/ paraganglial cells are normally absent [e.g., bone, liver, and lungs except in rare cases of primary hepatic and lung PGL]) and lymph nodes. Beyond its localizing value, molecular imaging also provides unique opportunities for better characterization of these tumors, mirroring ex vivo histologic classification but on the whole-body, in vivo, scale: ${ }^{123} \mathrm{I}-\mathrm{MIBG}$ or ${ }^{18} \mathrm{~F}-\mathrm{FD}$ opamine for the norepinephrine transporter system, somatostatin analogs labeled with ${ }^{68} \mathrm{Ga}$ for somatostatin receptor (SSR) expression, and 6-fluoro-3,4dihydroxyphenylalanine $/{ }^{18} \mathrm{~F}$-fluorodopa $/{ }^{18} \mathrm{~F}$-FDOPA for dopamine biosynthesis (2).

The second level of analysis relies on image-derived biomarkers (commonly known as radiomics). One of the great advantages of PET relative to other imaging modalities is its inherent quantitative nature. Traditionally, PET image analysis in the field of oncology applications has been restricted to the use of semiquantitative indices, such as SUV, representing the level of tumor activity concentration in the reconstructed images. In the setting of pheochromocytoma: SUV on ${ }^{18}$ F-FDOPA PET/CT correlates with urinary metanephrines and plasma chromogranin A (3). On ${ }^{18} \mathrm{~F}-$ FDG PET/CT, whole-body metabolic tumor volume and total lesion glycolysis were also found to be valuable predictors of biochemical response after cytoreductive surgery or ablative therapies for patients with PPGL (4).

Over the last few years, increasing interest has concentrated on the development of PET image segmentation algorithms that allow robust and reproducible determination of 3-dimensional tumor volumes from reconstructed PET images. Having accurately determined 3-dimensional functional tumor volumes allows the performance of further analysis, which can assess various other image-derived parameters including tumor shape and intratumor PET tracer distribution heterogeneity. The hypothesis behind the use of such PET image-derived parameters is their potential relationship with underlying biologic processes. This intratumor activity distribution heterogeneity may reflect, depending on the tracer used, different physiologic processes or any combination of them, including metabolism, proliferation, vascularization, hypoxia, necrosis, and 
apoptosis. This is of particular interest for PPGL because they often exhibit a heterogeneous appearance on imaging (2). Different approaches can be used to assess image-based heterogeneity. These include intensity histogram analysis, texture analysis, fractal analysis, or frequency-based methods. Hundreds of parameters evaluating intratumor activity distribution heterogeneity can be derived using such analysis. With hybrid imaging, further parameters derived from the CT or MRI component of the study may add further details to the tumor analysis. These parameters are characterized by variable levels of correlation and, under certain conditions, provide complementary information. Recently, the advent of robust machine-learning approaches may allow for a more robust parameter selection and combination than more traditional statistical analysis, albeit with a need for extensive validation and evaluation across imaging centers and patient populations. The role of radiomics in treatment personalization should be studied within several contexts such as for providing prognostic, predictive, and companion biomarkers. Finally, using radiomics to determine tumor-specific genetic profiles or alternatively combining imaging-based biomarkers with genomic information is an emerging field of investigation termed radiogenomics, clearly intersecting with the next level of analysis. These new developments are under technical and clinical evaluation in the setting of PPGL.

The third level of analysis (i.e., genotypic) is to translate imaging phenotypes into knowledge of disease mechanisms, especially their mutational and transcriptomic basis. For PPGL, both genotype and location drives imaging phenotype. The number of major drivers involved in the pathogenesis of PPGL are limited $(<15)$ and mutually exclusive. A majority of patients who have familial PGL have mutations in one of the SDH subunit genes (A-D, collectively named $S D H x$ ) that form the SDH enzyme complex, which is part of both the tricarboxylic acid cycle (TCA) cycle and the respiratory electron transport chain. SDHxrelated PPGL syndromes tend to have relatively indistinct clinical presentations across patients: PPGL, gastrointestinal stromal tumor, pituitary adenomas, and renal cell carcinoma all have variable penetrance across the different syndromes. SDHx-related PPGL also share common imaging features that distinguish them from other PPGL (5). Most PPGL exhibit moderate to low ${ }^{18} \mathrm{~F}-\mathrm{FDG}$ uptake whereas $S D H x$-related PPGL exhibit highly elevated ${ }^{18} \mathrm{~F}$ FDG uptake (6). Altered metabolism is a key feature of PPGLrelated SDHx mutations. In these tumors, TCA cycle blockade results in the accumulation of enormous concentrations of succinate (i.e., the substrate of SDH) that can be detected by in vitro and in vivo metabolomic studies.

Succinate is thought to be the main driver of ${ }^{18} \mathrm{~F}$-FDG phenotype in these tumors via a set of distinct intracellular and extracellular actions. Succinate, as some other oncometabolites, inhibits 2-oxoglutarate-dependent dioxygenases, which include members of the EglN1-3 (also called prolyl hydroxylases 1-3) family, and plays a key role in the regulation of the stability of hypoxia-inducible $\alpha$-isoforms. HIF- $1 \alpha$ and HIF- $2 \alpha$ exert a myriad of actions that promote tumorigenesis including stimulation of glucose transport (for HIF- $1 \alpha$ and HIF-2 $\alpha$ ) and glycolysis (for HIF- $1 \alpha$ only) via an upregulation of the expression of the glycolytic enzymes and PDK1. Elevated ${ }^{18}$ F-FDG accumulation can therefore be due to succinate-driven stabilization of HIF- $\alpha$ isoforms despite normal oxygen supply (also called pseudohypoxia), leading to a genetically determined Warburg effect (7). Another factor is related to the extracellular action of succinate on surrounding stroma cell carbohydrate metabolism via a hormonelike action (8). SDHxmutated PPGL of sympathetic origin also exhibit a less-well differentiated phenotype with respect to normal tissue function (moderate to low ${ }^{18} \mathrm{~F}$-FDopamine or ${ }^{123} \mathrm{I} /{ }^{131} \mathrm{I}-\mathrm{MIBG}$ ) than other subtypes, which has been suggested to be attributed to tumorigenesis arising for less differentiated progenitors. This is not the case for head and neck PGL, which remain well differentiated even at later stages. Finally, SDHx-related PPGL overexpress SSRs and are therefore targetable with somatostatin analogs (SSAs) labeled with diagnostic radionuclides (e.g., ${ }^{68} \mathrm{Ga}-\mathrm{SSA}$ ). The biologic function of SSRs in chromaffin cells remains unclear. PPGL that carry EPAS1 (i.e., HIF2A) mutations do not express an $S D H$-like phenotype (but have high ${ }^{18} \mathrm{~F}$-FDOPA uptake and moderate to low tumoral uptake on ${ }^{68} \mathrm{Ga}-\mathrm{SSA}$ ), despite stabilization of HIF- $2 \alpha$ potentially contradicting the previous hypotheses that succinate represents the main connecting hub between SDHx imaging phenotype and genotype, and suggesting that other factors may be at play. Other PPGL usually exhibit low to moderate ${ }^{18} \mathrm{~F}$-FDG uptake, high ${ }^{18} \mathrm{~F}$-FDOPA uptake, high to low ${ }^{18} \mathrm{~F}$-FDopamine uptake depending on their secretory profile (negative in head and neck paraganglioma vs. positive in chromaffin cell-derived tumors) and high ${ }^{68} \mathrm{Ga}-\mathrm{SSA}$ uptake (2).

The fourth level of analysis is more conceptual: aiming to advance and expand the nosologic classification of cancers. This requires the integration of information from multiple domains but particularly with clinical behavior and outcomes. In medicine, several studies have shown that ${ }^{18} \mathrm{~F}$-FDG uptake was dependent on the tumor mutational status, and now being readily available in many clinical settings, this may provide a useful first step in defining tumor biology. The unique opportunity for understanding PPGL is the opportunity to apply different radiopharmaceuticals to fully characterize tumors at a molecular level. A comprehensive molecular classification of these tumors lea to discoveries of unique clusters related to these tumors (9). The clusters are now linked to individual functional imaging modalities that find that, for example, $S D H x$ are optimally localized by ${ }^{68} \mathrm{Ga}$-SSA PET/CT and EPAS1- and MAX-related PGL by ${ }^{18} \mathrm{~F}-$ DOPA PET/CT. Furthermore, new data related to norepinephrine as a significant risk factor in the development of metastatic disease and previously published mechanisms that norepinephrine shuffles glucose into cells via $\alpha 1$-adrenoceptor suggests the role of ${ }^{18} \mathrm{~F}$-FDG application in metastatic PPGL. In the near future, new metabolomic data will undoubtedly uncover the new contribution of various metabolites in unique functional imaging signatures of these tumors. Systems biology and "big data" have key roles in such analyses but are challenged in the case of PPGL by the rarity of each of the different subgroups based on genomic characterization. In this context, the information provided by multiparametric molecular imaging in individual patients may represent an important asset for precision medicine, allowing for the identification of varied imaging phenotypes that may reflect differing genomic profiles of individual lesions (10) arising as a result of clonally constrained mutations due to branched evolution within the primary tumor, spontaneous metastases during growth, or following the selective pressure of treatment. By demonstrating such heterogeneity, their genomic basis could be interrogated by a multisite molecular imaging-guided biopsy.

\section{CONCLUSION}

Although the topic of imaging, including nuclear medicine, has been focused on the accurate detection of lesions ("lumpology"), increasingly it is being recognized that molecular imaging provides a unique opportunity for biologic characterization of disease on a wholebody scale through identification of phenotypic heterogeneity. In 
this role, it complements all elements of the pathologic assessment armentarium including histology, molecular pathology, genomics, and transcriptomics (1). Clearly, the big data embedded in the imaging dataset requires a more sophisticated analysis and a move beyond a simple visual interpretation or semiquantitative parameters such as the SUV of a few lesions. Whole-body segmentation methods facilitating the extraction of multiple image-based biomarkers combined with detailed radiogenomic analysis using machine-learning algorithms linked to richly annotated clinical data will aid our planning of optimal management in individual patients (e.g., diagnosis, survival, tumor evolution, decision). This is a key goal in the setting of rare diseases for which an evidence base from large clinical trials is difficult or impossible to create, as well as our understanding of complex diseases such as PPGL, which have uncertain clinical behavior and highly variable prognosis.

\section{DISCLOSURE}

No potential conflict of interest relevant to this article was reported.

\section{REFERENCES}

1. Hofman MS, Hicks RJ. Moving beyond "lumpology": PET/CT imaging of pheochromocytoma and paraganglioma. Clin Cancer Res. 2015;21:3815-3817.
2. Taïeb D, Hicks RJ, Hindie E, et al. European Association of Nuclear Medicine practice guideline/Society of Nuclear Medicine and Molecular Imaging procedure standard 2019 for radionuclide imaging of phaeochromocytoma and paraganglioma. Eur J Nucl Med Mol Imaging. 2019;46:2112-2137.

3. Amodru V, Guerin C, Delcourt S, et al. Quantitative ${ }^{18} \mathrm{~F}-\mathrm{DOPA}$ PET/CT in pheochromocytoma: the relationship between tumor secretion and its biochemical phenotype. Eur J Nucl Med Mol Imaging. 2018;45:278-282.

4. Patel D, Mehta A, Nilubol N, Dieckmann W, Pacak K, Kebebew E. Total ${ }^{18}$ FFDG PET/CT metabolic tumor volume is associated with postoperative biochemical response in patients with metastatic pheochromocytomas and paragangliomas. Ann Surg. 2016;263:582-587.

5. Crona J, Taieb D, Pacak K. New perspectives on pheochromocytoma and paraganglioma: toward a molecular classification. Endocr Rev. 2017;38:489-515.

6. Taieb D, Pacak K. New insights into the nuclear imaging phenotypes of cluster 1 pheochromocytoma and paraganglioma. Trends Endocrinol Metab. 2017;28:807817.

7. van Berkel A, Rao JU, Kusters B, et al. Correlation between in vivo ${ }^{18}$ F-FDG PET and immunohistochemical markers of glucose uptake and metabolism in pheochromocytoma and paraganglioma. J Nucl Med. 2014;55:1253-1259.

8. Garrigue P, Bodin-Hullin A, Balasse L, et al. The evolving role of succinate in tumor metabolism: an ${ }^{18}$ F-FDG-based study. J Nucl Med. 2017;58:17491755 .

9. Fishbein L, Leshchiner I, Walter V, et al. Comprehensive molecular characterization of pheochromocytoma and paraganglioma. Cancer Cell. 2017;31:181-193.

10. Flynn A, Dwight T, Benn D, et al. Cousins not twins: intratumoural and intertumoural heterogeneity in syndromic neuroendocrine tumours. J Pathol. 2017; 242:273-283. 